\title{
Editorial
}

\section{Exercise Medicine: An International Open Access Journal Covering the Entirety of Sports and Exercise Medicine}

\author{
Junghoon $\mathrm{Kim}^{*}$ \\ Editor-in-Chief, Department of Preventive Medicine, Gachon University College of Medicine, Incheon, Korea
}

\begin{abstract}
I am pleased that Exercise Medicine (EM) has entered its second year of publication, and I would like to welcome the readers, contributors, and Editorial Board members as the first editor-in-chief. EM is a new, international, open access and peer-reviewed, multidisciplinary scholarly journal of scientific studies related to all aspects of sports and exercise medicine. Our aim is to bridge the gap between research and practice in the fields of exercise, health promotion, disease prevention and treatment, and human performance and health. EM has a special focus on definitive articles that interpret and evaluate the current literature to provide the rationale for, and application of, research findings for exercise specialists and physiologists, public health specialists, physicians, physical therapists, and trainers. The journal publishes research articles, clinical studies, case studies, and brief reports on current topics in sports and exercise medicine. $E M$ also welcomes the submission of high-quality review articles and meta-analyses in the above fields.

Our goal is to offer an academic platform of high quality and peer-reviewed articles that can disseminate information for the members of our research community. All relevant high-quality and innovative papers for sports and exercise medicine are welcome, and, will be published after a rigorous peer-review procedure, if accepted. We also aim to process manuscripts quickly and publish them shortly after the peer-review procedure. Recent high-quality papers have been peer-reviewed and published within 4 weeks of submission.
\end{abstract}

*Correspondence: Junghoon Kim, Department of Preventive Medicine, Gachon University College of Medicine, Incheon 21936, Republic of Korea, 155 Gaetbeol-ro, Yeonsu-gu, Incheon 21999, Republic of Korea.

E-mail address: junghoonkim@gachon.ac.kr

Received: March 8, 2018; Accepted: March 19, 2018;

Published online: March 19, 2018
The Editorial Board comprises a diverse group of experts that reflect the broad range of topics covered by the journal and the varied interests of the research community it represents. The success of this endeavor depends on the journal's ability to convince this community of the value of this contribution. In addition, this value will depend on the high-quality articles that we encourage you and your colleagues to submit. The members of the Editorial Board look forward to your contributions.

We invite investigators with an interest in the field of sports and exercise medicine to contribute their original research articles to this journal. Recently, we have published articles on various topics, including disease prevention and treatment by exercise [1,2], exercise and physical activity for health promotion [3], injury prevention and rehabilitation in sports [4], and recommendations for physical activity and nutrition [5].

Researchers from academic institutions and research personnel from corporate research departments are welcome to contribute research articles to this new open access journal. This will enable it to share the latest research results within the research community without any barriers. We hope that this journal will aid interdisciplinary collaboration and networking between exercise/public health specialists and scholars in the academic, policy and clinical fields. We aim to inspire and facilitate more global collaborations through studies that will be published in EM.

Fields of particular interest to the journal include (but are not limited to):

- Sports and Exercise Medicine

- Rehabilitation

- Exercise Physiology

- Biochemistry

- Health Promotion 
- Physical Activity for Children

- Epidemiology

- Aging

- Disease Management

- Human Performance and Health

\section{Conflicts of interest}

The authors declare no conflict of interest.

\section{ORCID}

Junghoon Kim (http://orcid.org/0000-0003-4802-010X)

\section{REFERENCES}

1. Hart PD. Using structural equation modeling to examine the effects of sex and physical activity on the metabolic syndrome and health-related quality of life relationship. Exerc Med. 2018; 2:3.

2. Oh S, Han G, Kim B, Shoda J. Regular exercise as a secondary practical treatment for nonalcoholic fatty liver disease. Exerc Med. 2018; 2:4.

3. Benavidez G, Hart PD. Effects of yoga on measures of health-related quality of life from sf-36 and sf- 12 assessments: A systematic review and meta-analysis. Exerc Med. 2017; 1:5.

4. An KO. Exercise rehabilitation after anterior cruciate ligament reconstruction. Exerc Med. 2017; 1:1.

5. Kim J. Longitudinal trend of prevalence of meeting physical activity guidelines among korean adults. Exerc Med. 2017; 1:2. 